\title{
Unmanned: dimensão cívica e política de um videojogo
}

\author{
Lucinda Saldanha, Marta Pinto \\ \& Pedro D. Ferreira
}

\begin{abstract}
Resumo
O estudo dos videojogos sérios tem vindo a crescer nas últimas décadas, apontando para o potencial dos videojogos como meios digitais importantes para a aprendizagem da cidadania e da participação cívica e política. Este artigo apresenta o estudo de caso de um ciclo de experimentação e de reflexão sobre o videojogo sério "Unmanned", no contexto de três oficinas sobre o uso educativo de videojogos no ensino superior, que decorreram nos meses de Maio e Junho de 2017. Apresentam-se os resultados de uma análise temática, desenvolvida a partir de um conjunto de categorias relativas à compreensão da dimensão cívica e política em videojogos. Os resultados reforçam o potencial deste videojogo para a aprendizagem da cidadania e participação cívica e política, e dos jogos sérios como estratégia para aprender e educar, de forma crítica e transformativa.
\end{abstract}

\section{Palavras-chave}

videojogos sérios; cidadania; dimensão cívica; dimensão política. 


\section{Unmanned: political and civic dimension of a videogame}

Abstract: The study of serious games has been growing in recent decades and pointing to the potential of video games as an important digital media for learning citizenship and civic and political participation. This article presents the case study of the serious game "Unmanned", object of an experimentation and reflection cycle, in the context of three workshops on the educational use of video games in higher education that took place in May and June 2017. The results of a thematic analysis, developed from a set of categories related to the understanding of the civic dimension in video games, are presented. Results reinforce the potential of this video game in terms of learning citizenship and civic and political participation, and of the use of serious games as a strategy to teach and learn in a critical and transformative manner.

Key words: serious games; citizenship; civic dimension; political dimension.

\section{Unmanned: dimension politique et civique du jeu vidéo}

Résumé: L'étude des jeux vidéo sérieux a pris de l'ampleur au cours des dernières décennies, soulignant le potentiel des jeux vidéo en tant que moyen numérique important d'apprentissage de la citoyenneté et de la participation civique et politique. Cet article présente l'étude de cas d'un jeu sérieux «Unmanned», cible d'un cycle d'expérimentation et de réflexion, dans le cadre de trois ateliers sur l'utilisation pédagogique des jeux vidéo dans l'enseignement supérieur, qui ont eu lieu en mai et juin 2017. Les résultats d'une analyse thématique, développée à partir d'un ensemble de catégories liées à la compréhension de la dimension civique dans les jeux vidéo, sont présentés. Les résultats renforcent le potentiel de ce jeu vidéo pour apprendre la citoyenneté et la participation civique et politique, et celui des jeux sérieux comme stratégie pour apprendre et éduquer, d'une manière critique et transformatrice.

Mots-clés: jeux vidéo sérieux; citoyenneté; dimension civique; dimension politique.

\section{Unmanned: dimensión cívica y política de un videojuego}

Resumen: El estudio de los videojuegos serios ha crecido en las últimas décadas, apuntando al potencial de los videojuegos como medios digitales importantes en el aprendizaje de la ciudadanía y de la participación cívica y política. Este artículo presenta el estudio de caso de un ciclo de experimentación y de reflexión sobre el videojuego serio "Unmanned", en el contexto de tres talleres sobre el uso educativo de los videojuegos en la enseñanza superior, que se desarrollaron entre los meses de mayo y junio de 2017. Presenta los resultados de un análisis temático, desarrollado a partir de un conjunto de categorías relativas a la comprensión de la dimensión cívica y política en los videojuegos. Los resultados refuerzan el potencial de este videojuego en el aprendizaje de la ciudadanía y participación cívica y política, y de los juegos serios como estrategia para aprender y educar, de una forma crítica y transformativa.

Palabras clave: videojuegos serios; ciudadanía; dimensión cívica; dimensión política. 


\section{Introdução}

A linha de investigação que cruza videojogos sérios com questões de cidadania e da sua aprendizagem tem vindo a crescer nas últimas décadas, apontando para o potencial dos videojogos como meios digitais importantes para a aprendizagem da cidadania e da participação cívica e política (Frasca, 2001, Bogost, 2006; Mitgutsch \& Alvarado, 2012). Vários são os autores que têm estudado o impacto da digitalização da cultura na forma como conceptualizamos, perspetivamos e vivemos a educação e a cidadania (Bennett, 2008; Castells, 2011).

De facto, os jogos têm sido referidos como estratégias privilegiadas de aprendizagem de comportamentos, atitudes, formas de ser e de estar, e também de participação e de criação de cultura (Huizinga, 1971), que, na combinação entre o analógico e o digital, se têm cruzado, nas últimas décadas, para criar novos contextos de aprendizagem e de participação cívica e política e novas possibilidades de trabalho em rede, segundo o que Jenkins et al (2009) tem designado de "participatory digital cultures".

Nesta linha, tem surgido uma nova área de investigação, englobando a "Digital Media and Democracy" e a Cidadania Digital, que se tem dedicado a estudar o potencial destes novos media, enquanto instrumentos para o aprofundamento da democracia e para a mudança social, e enquanto espaços emergentes de participação cívica e política. De facto, os videojogos como medium digital têm sido estudados como um novo movimento social, à escala global, pela quantidade de pessoas envolvidas e pelas múltiplas possibilidades de atuação e de influência que têm vindo a revelar. Estes impactos ainda se encontram a ser estudados e problematizados e é neste contexto que este artigo se situa: como é que estes novos meios digitais nos provocam a compreender e a recriar o significado e o sentido da educação e da cidadania, e como nos trazem novas ferramentas para aprender e educar, de forma crítica e transformadora (Freire, 2014).

Especificamente o conceito de jogo sério é aquele que mais se aproxima dos propósitos de uma educação para o desenvolvimento sociopolítico. É emergente a linha de investigação que estuda a forma como os videojogos sérios se relacionam com uma educação emancipadora e transformadora, explicitando como estes potenciam, de múltiplas formas, uma aprendizagem significativa e criadora de sentido, lúdica, ativa, situada, experiencial, auto-regulada e personalizada (Gee, 2009; Becker, 2017) e como desafiam a ação sobre questões significativas para os/as aprendentes e comunicam com o seu sentido de identidade, com efeito significativo ao nível do empoderamento e do seu sentido de controlo (Gee, 2009; Squire, 2011).

Como os videojogos apelam a experiências de flow, embodiement, experiências de relação afetiva e emocional, nas quais diferentes dimensões da identidade estão presentes, já vários estudos têm analisado o seu potencial para o desenvolvimento de 
uma aprendizagem com sentido e significado para os/as aprendentes e como facilitadores da mudança que este tipo de aprendizagem provoca. Neste sentido, os estudos que incluem videojogos socorrem-se mais vezes de modelos de aprendizagem construtivistas e humanistas, que advogam uma aprendizagem ativa e experiencial (Becker, 2017) e ciclos continuados de ação e de reflexão (Freire, 2017).

Em Portugal, esta linha de investigação ainda se encontra a dar os primeiros passos e ainda há poucos estudos que caracterizem a relação dos/as estudantes e docentes com os videojogos sérios e que apresentem os seus usos, quer para fins educativos quer para fins cívicos e políticos, no contexto de ensino superior. Os estudos existentes focam sobretudo os hábitos de jogo e as perceções sobre videojogos e evidenciam altas taxas de uso e de experiência com videojogos, quer de estudantes quer de docentes, fora do contexto de sala de aula, e percentagens ainda baixas de uso neste contexto. Contudo, estudantes e docentes apresentam perceções favoráveis relativamente ao seu uso com fins educativos, compreendendo a mobilização do seu potencial para aprender e educar (Oliveira \& Pessoa, 2008; Pinto \& Ferreira, 2017).

Num mundo onde os recursos digitais se apresentam como ubíquos, e no qual os estudantes do ensino superior vivem rodeados de experiências digitais, urge caracterizar qual o seu uso, quer ao nível de experiência de utilização, quer ao nível da experiência de criação. No ensino superior tem-se tentando compreender como os videojogos sérios, enquanto um tipo de tecnologia educativa emergente, têm sido utilizados em combinação com outras ferramentas, e quais os métodos de ensino-aprendizagem que permitem explorar dimensões cívicas e estimular as práticas de cidadania. Interessa conhecer de que forma os contextos digitais vão influenciando o desenvolvimento cívico e sócio-político de jovens adultos no ensino superior e quais as ferramentas que podem ser usadas para o estímulo deste desenvolvimento, num momento de vida que poderá ser propício ao seu compromisso, participação e ação cívica (White, 2013).

Internacionalmente, a ideia de que os videojogos sérios têm ou podem ter influência no desenvolvimento cívico e de uma educação socio-política tem feito surgir novos conceitos que associam os videojogos à dimensão da cidadania e da mudança social: "videojogo cívico e político", "games for change", "games for good" ou "impact games" (Khane, Middaugh \& Evans, 2008). Estes conceitos acentuam a valorização de processos de aprendizagem e de mudança provocados pela experiência de jogar videojogos, designados como "transformational play", o "jogo transformador", com potencial para criar e questionar narrativas de sentido já existentes (Bogost, 2006).

$\mathrm{Na}$ mesma linha, encontramos estudos que analisam o conceito de conscientização de Freire e o trabalho de Augusto Boal, na sua relação com os videojogos sérios: "games for the oppressed", demonstrando como é que as estratégias de conscientização podem ser aplicadas na (e através) da experiência e criação de videojogos de simulação da vida real e de narração de histórias, para aumentar o pensamento cívico 
e crítico, a capacidade de leitura e de construção da realidade, de exploração e criação de sentido (Frasca, 2001).

O foco no potencial de criação de videojogos para a construção de outras narrativas e para a criação colaborativa de mudança social, ainda se encontra pouco explorado internacionalmente (Bogost, 2006). No entanto, têm surgido estudos sobre o potencial do uso da narrativa, de storytelling, da recriação de histórias e situações reais de vida da comunidade, com recurso a videojogos (Frasca, 2001), que sugerem que o processo de criação de videojogos pode ser promotor de desenvolvimento sócio-político.

\section{Dimensão cívica e política em videojogos}

Neste entrecruzamento entre videojogos sérios e cidadania, em contexto internacional, é possível encontrar estudos que nos permitem realizar uma leitura compreensiva e analítica dos videojogos sérios, nas suas dimensões cívica e política. Estes estudos serviram como base de uma revisão e integração da literatura, da qual resultaram as quatro categorias principais, apresentadas abaixo:

\section{1) Conhecimento cívico e político:}

Informação: apresentação/exposição e/ou desafio à procura ativa de conteúdos, factos, notícias, relatos históricos relacionados com dimensões políticas, sociais e cívicas, disponíveis em diferentes formatos, que possibilitará a aquisição de conhecimentos nestas áreas de estudo.

Direitos e Deveres: apresentação de informação concreta e/ou capacidade de representação do funcionamento do sistema político, instituições, governo e da democracia, bem como sobre os direitos e deveres de um/a cidadão/cidadã.

Exposição a diferentes/múltiplas perspetivas: apresentação de diferentes perspetivas e de diferentes posições ideológicas ou apresentação de uma visão unidirecional e uma única possibilidade, fechada e rígida de olhar a realidade ou de a apresentar.

(Bogost, 2006; Flanagan, 2007; Khane et al., 2008; Bers, 2010; Connolly et al., 2012; Mitgutsch \& Alvarado, 2012).

\section{2) Desafios éticos}

Dilemas: apresentação de dilemas- situações de confronto com a necessidade de posicionamento, com base em valores e princípios éticos.

Consciência ética: processo de identificação, análise e interpretação de situações de natureza ética, para criação de um posicionamento ético sobre as estruturas e regras morais e éticas pessoais, culturais e sociais. Entendimento sobre as 
possibilidades de questionamento, alteração, mudança ou de repetição de normas e princípios morais e éticos. Exposição (ou não) a diferentes códigos éticos.

Decisões: processo de tomada de decisões, que implicará a identificação, reflexão e compreensão de valores, de crenças e de posições ideológicas, bem como das estratégias diversificadas de tomada de decisão, tendo em conta o confronto (ou não) com as consequências.

(Khane et al., 2008; Bers, 2010; Stevenson, 2011; Bogost, 2012; Schrier, 2015).

\section{3) Interação social:}

Outros - capacidades de relação e diferença: possibilidades de desenvolvimento de competências sociais e relacionais (empatia, gestão de emoções, tomada de perspetiva do Outro, trabalho em equipa, resolução e mediação de conflitos), e de exposição à diversidade e à diferença.

Identidades e Papéis: apresentação de oportunidades de exploração e de experimentação de diferentes identidades e papéis.

Poder: exposição e apresentação de diferentes formas e de diferentes relações de poder, bem como de representações e categorias sociais.

(Khane et al., 2008; Barab, 2010; Bers, 2010; ; Mitgutsch \& Alvarado, 2012; Schrier, 2015).

\section{4) Ação cívica e política:}

Atitudes: potencial e predisposição para agir, orientação e motivação para o envolvimento em ações cívicas e políticas.

Ligação ao mundo fora do Jogo/práticas e competências de cidadania: apresentação e/ou exposição, exploração e experimentação de competências e práticas de cidadania, com ligação e transferência para a realidade do mundo fora do jogo.

Agência/Estrutura: presença do binómio agência (capacidade de transformação, mudança, liberdade para criar, dentro e fora do jogo) e estrutura (condicionamento de regras, dentro de uma forma única de organização e funcionamento, no jogo e fora do jogo).

(Flanagan et al., 2007; Khane et al., 2008; Bennett et al., 2009; Raphael et al., 2010).

\section{Apresentação do videojogo Unmanned}

Unmanned é um videojogo criado por Jim Munroe e desenvolvido pela Molleindustria, no ano de 2012 (ver figura 1). O videojogo, apresentado como uma instalação de arte semi-jogável, faz parte do género subversivo de jogos Molleindustria, que tem como um dos seus principais objetivos contribuir para um questionamento político e reflexivo sobre 
a indústria dos videojogos e o impacto da cultura de jogo na sociedade contemporânea. Este videojogo recebeu várias menções honrosas na imprensa e vários prémios, especificamente dois prémios no "Games for Change Festival", no ano em que foi criado.

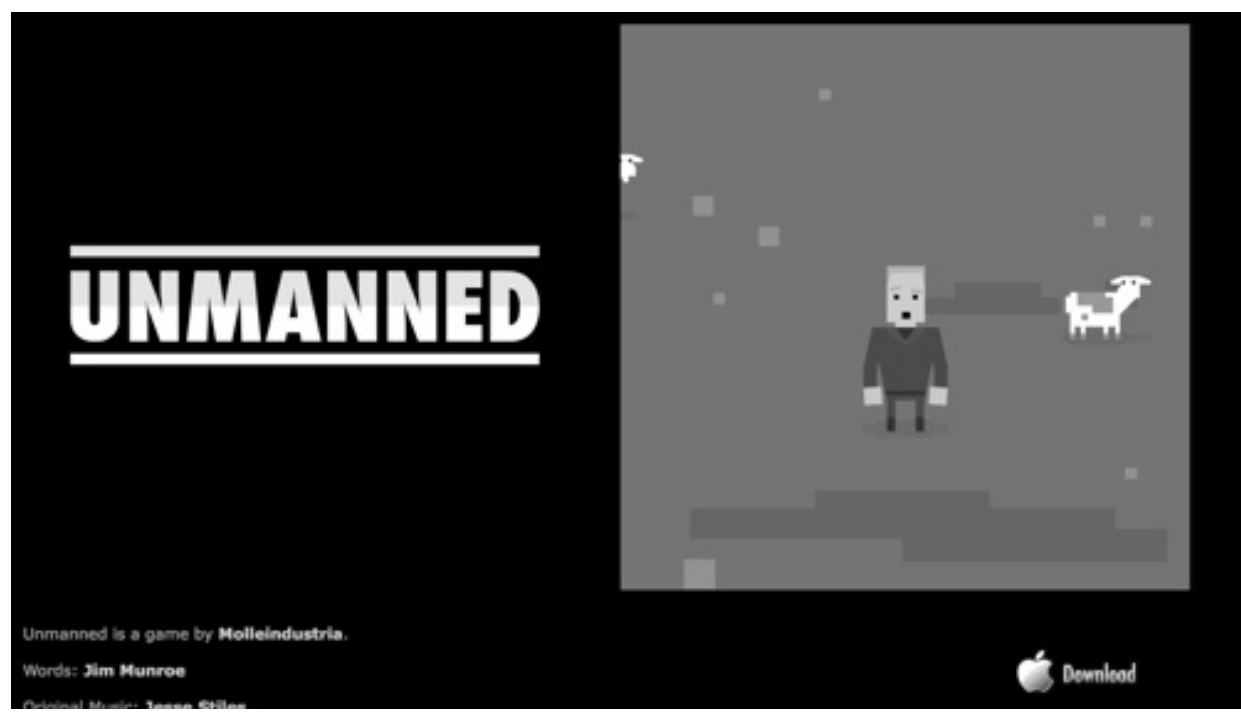

Figura 1: imagem do primeiro ecrã do videojogo Unmanned, acedida a partir de http:// unmanned.molleindustria.org

A premissa principal do jogo tem como base uma narrativa escrita e visual e uma simulação satírica, centrada na personagem de um piloto de drones de guerra à distância. O jogo permite-nos experimentar e explorar a "rotina diária normal" e os vários papéis sociais de um piloto de drones (pai, marido, colega de trabalho), os seus conflitos internos privados, reconhecendo a existência e dando visibilidade a uma guerra que é feita à distância ou paradoxalmente muito próxima da nossa vida quotidiana, criticando o suposto "herói de guerra" e amplificando temáticas como a anomia, a alienação e a sociedade fragmentada.

O jogo apresenta e identifica, de forma crítica e irónica, os novos métodos de guerra utilizados, desafiando um questionamento ético sobre as ações diárias concretas, que envolvem esta forma de "guerra tecnológica", e os paradoxos dos elementos de guerra que muitos jogos apresentam. Ao longo do jogo, o/a jogador/a é confrontado/a com um conjunto de cenários provocativos sobre os quais é desafiado/a a tomar uma posição, a fazer escolhas significativas, a co-criar a narrativa e a expor-se às suas consequências. O jogo é jogado apenas com o rato do computador, exigindo alguma coordenação motora, e o investimento em múltiplos mini-jogos, pois o/a jogador/a 
é confrontado/a com ecrãs duplos e divididos. Apresenta uma música de fundo, em segundo plano, que revela um cenário emocional tendencialmente frio e melancólico.

\section{Metodologia}

Nas oficinas, foi utilizada uma metodologia ativa e participativa, que considera a importância da voz única de cada participante, com a qual se procurou valorizar as experiências de vida e os conhecimentos provenientes de várias áreas, e que buscou ativamente a construção conjunta de conhecimento como processo de co-criação (Chambers, 2002; Konings et al., 2007; Khaled \& Vasalou, 2014).

Os/as participantes das oficinas organizaram-se em pares para jogar o videojogo proposto (entre outros não explorados neste artigo). Foi-lhes pedido que jogassem o jogo Unmanned durante 25 minutos. Após este tempo, foi pedido que partilhassem em grande grupo as suas perceções sobre a experiência de jogo. O discurso dos/as participantes foi recolhido através de gravação áudio e vídeo e posteriormente transcrito. Com base nestas transcrições foi realizada uma análise temática, utilizando como base de análise as categorias e subcategorias apresentadas para a dimensão cívica e política de videojogos.

\section{Amostra}

O videojogo Unmanned foi experienciado em três oficinas (uma sessão por oficina), com grupos mistos de estudantes, professores/as e investigadores/as universitários/ as de várias áreas de estudo do ensino superior. As oficinas tiveram 30 participantes (cada oficina com uma média de 10 participantes), com idades entre os 19 e os 48 anos, 16 mulheres e 9 homens. As oficinas tiveram a duração total de 12 horas cada, e integraram ciclos de ação de uso e experiência de jogo, e de reflexão sobre a experiência de jogar, e decorreram entre os meses de Maio e Junho de 2017.

\section{Resultados e Discussão de Resultados}

Nesta secção apresentamos os resultados da análise temática, por categoria de análise.

\section{Categoria conhecimento cívico e político}

Os resultados indicam que este videojogo favorece a tomada de consciência, o conhecimento e a reflexão crítica sobre o sistema social, político e cívico: "este jogo era totalmente uma crítica social. Este jogo acaba por gozar com o estereótipo americano e ao mesmo tempo é existencial... e faz-nos pensar se é possível tentar mudá-lo ou 
não" (participante J); "acho que mais do que ironia, era mesmo uma sátira da sociedade, de como vivemos" (p. E).

Este videojogo facilitou a caracterização crítica de um modelo de sociedade, das suas diferentes formas de representação, do poder e influência das ideologias sobre o comportamento e a consciência individual: "O jogo apresenta-nos uma determinada representação da realidade e coloca-nos perante ela: podemos questioná-la, criá-la. É uma possibilidade de representação da realidade" (p. L).

Ao mesmo tempo, fica patente para alguns/as participantes a vivência do videojogo como experiência e não com um meio instrumental, para atingir um fim ou um resultado explícito: "este é o tipo de jogo em que eu preciso de me deixar levar mais por essa experiência. Experimentar o jogo não no sentido de ganhar mais ou menos, mas no sentido de perceber até onde eu vou com aquilo" (p. E).

O videojogo parece potenciar oportunidades de reflexão sobre as possibilidades de representação e de construção da realidade social e política: "o jogo proporciona-nos reflexão, pensarmos no mundo em que vivemos (p. J); "Eu senti-me a refletir muito mais neste jogo. Logo que comecei a jogar, senti-me logo a refletir sobre a temática sobre a qual estava a ser exposta e não estive muito importada em ganhar pontos, porque estava numa atitude de exploração. Foi como eu vivi isto" (p. E).

Embora os participantes reforcem que esta reflexão necessita de ser estimulada no contexto em que é apresentado o videojogo e que este funciona como um estímulo para a reflexão e aprendizagem subsequente: "tudo o que pode acontecer de aprendizagens é o insight que se pode fazer depois disso, mas resta saber se o utilizador está disponível para pensar nisso. Estamos a ver o jogo como um processo... "(p. I); "é preciso que os jogadores tenham algumas âncoras a que se agarrar e às quais pudessem prender essa reflexão, sob pena de acontecer o desligamento da realidade" (p. I).

Os/as participantes assumem que a dimensão estética, designadamente a linguagem musical, produzem climas e atmosferas favoráveis e propícias a processos reflexivos: "acho muito importante esta parte estrutural do jogo, porque uma música de fundo, no início, mais séria, condiciona-nos a que seja uma coisa séria, não nos leva a "vamos matar toda a gente". Há músicas que nos ajudam a entrar na parte reflexiva" (p. J).

Assim como a própria mecânica do videojogo poderá estimular à reflexividade: "não é só uma questão de descobrir ou descodificar o jogo, porque isso é que leva à reflexão. Posso jogar só por exploração mecânica do jogo, mas neste jogo, acho que a exploração mecânica me leva à reflexão" (p. J).

Os/as participantes referem que este videojogo estimula a reflexão sobre diversas temáticas cívicas e políticas, contribui para o alargamento do conhecimento sobre contextos sociais e referem ter ganho conhecimento em áreas associadas, como os direitos humanos, a geografia e a gestão de recursos. 


\section{Categoria desafios éticos}

O videojogo parece favorecer sentimentos de incerteza e de ambivalência, na tensão entre estrutura e liberdade para explorar, e um contexto propício à dissonância cognitiva e questionamento crítico: "não estou habituada a fazer coisas que não sel para que servem, nem o que é suposto fazer. Tenho de andar o tempo todo à sorte e ver o que acontece, para ver se percebo o que é suposto eu fazer. Não gosto de lidar com a incerteza... isso irrita-me mais do que me diverte" (p. M).

Pelo contrário, para outros/as participantes, este sentimento de incerteza é associado ao prazer da descoberta, ao entusiasmo da exploração e é considerado um estímulo: "O jogo era muito confuso e tinha piada por isso, por não saber o que estamos a fazer ou onde ia dar" (p. S).

Estas diferentes reações foram associadas pelos/as participantes a distintos estilos de aprendizagem e a diferentes experiências prévias relacionadas com diferenças geracionais: "umas pessoas gostam mais de segurança e de certeza e outras gostam de aprender pela descoberta. Isso depende também da idade e da experiência. Acho que os mais novos se saem melhor à descoberta e nós precisamos de mais instruções" (p. M).

O tom irónico e o humor presente no videojogo acentuam e reforçam positivamente, para alguns/as participantes, esta ambivalência e o "sentimento de estarmos a calcar terreno movediço" (p. I).

De facto, os/as participantes reforçam mesmo que o videojogo pode criar intencionalmente no jogador sentimentos de incerteza: "(o jogo) pode ter o objetivo de não ter objetivos e de provocar-nos intencionalmente sentimentos de incerteza, para nos permitir aprender a lidar com eles ( $p$. A).

Contudo, alguns/as participantes advogam um equilíbrio entre o enquadramento e a liberdade de exploração: "(...), mas se fica demasiado livre essa exploração fica avulsa, e se fica avulsa, não leva (os/as jogadores ou estudantes) para os pontos onde eu os quero levar" (p. I).

Alguns/as participantes advogam mesmo a importância da exploração livre sem um enquadramento inicial para potenciar a liberdade imaginativa: "Estou a pensar que a exploração livre é muito interessante em termos de apropriação. Eu não sei nada sobre o jogo e a minha imaginação e apropriação é maior do que se eu já tiver um enquadramento, pois já condiciona a leitura" ( $p$. E).

Neste jogo fica patente, de forma irónica, a tensão entre a necessidade de ter objetivos claros e definidos num videojogo, numa lógica linear e objetiva, de uma certa linha de produção e eficiência: "fico satisfeito por cumprir sempre os objetivos do jogo", a par de uma lógica mais aberta, que possa abrir novas possibilidades de conhecer, de criar e de jogar, na tolerância de estados de ambiguidade, de um certo absurdo e de 
não saber: "não há plano e nem tudo pode ser planeado... não sabemos onde poderá levar-nos" (p. E).

Ao mesmo tempo, os dados indicam que este videojogo parece incentivar ativamente a experiência e exploração, em contexto seguro, de diversos comportamentos morais, incentivando a criação de narrativas alternativas, com base no questionamento das (não) consequências: "tive a oportunidade de experimentar "ser bom" e "ser mau" (p. L).

Assim como incentivar a modelação ou não do comportamento, através da consciência das consequências apresentadas pelo videojogo: "há um incentivo a experimentar outras possibilidades: não ser sempre cordial..." (p. H).

De facto, os/as jogadores/as são confrontados/as de forma permanente com escolhas (entre três opções), que os/as direcionam para cenários e possibilidades de narrativa diferentes. Os/as participantes sugerem que estas escolhas apresentam um carácter ético e moral, e encontram-se relacionadas com vários papéis de vida. Alguns/as participantes referem-se a estas escolhas, como a dilemas éticos: "sentia-me colocado numa situação de dilema: sentia-me envolvido emocionalmente com a minha decisão, e responsável pelas minhas ações" (p. M); "havia também valores, decisões éticas e desafios que emergiam do jogo e nos impulsionavam sempre a pensar no que fazer" (p. HE)

Acrescentam que estes dilemas deverão estar relacionados com a realidade para que possam fazer a diferença na aprendizagem de comportamentos cívicos: "estes jogos devem ter problemas reais. Para terem efeitos na cidadania devem ter alguma ligação prática com as nossas vidas" (p. T).

Embora vários/as participantes se tenham referido à autonomia que as escolhas sugerem na criação do próprio jogo, o tempo necessário para realizar escolhas ponderadas e sensatas e o processo de tomada de decisões importantes na vida é uma temática que o videojogo estimula à reflexão: "Neste jogo, vejo uma análise muito subjetiva: é sobre como se escolhe, para que se escolhe. Acho que é uma análise que cada pessoa tem de fazer por si mesma" (p. J); "é como se estivéssemos com muitos estímulos e tivéssemos de tomar uma decisão muito importante numa fração de segundos, pois se não o fizermos algo de mal pode acontecer" (p. O).

A par disso, o jogo parece ironizar as consequências sociais e visíveis das decisões e feitos do personagem, através da apresentação de medalhas, de forma quase indiscriminada: "Eu acho que neste jogo, a questão das medalhas é uma sátira à questão de ganhar medalhas. Podíamos ser bons ou maus. Era uma forma de gozar com a acumulação de medalhas que se procura ter, tanto nos jogos, como noutros contextos. Porque o grande objetivo dos jogos é alcançar objetivos- o achievement. E recebe-se medalhas por coisas que não são necessariamente boas ou más ou úteis. E acho que era um bocado criticar isso" (p. J). 
Estas experiências conduziram à reflexão sobre as práticas de reforço ou de feedback nos videojogos e na vida fora do jogo: "simplesmente não havia consequências. Às vezes, recebia-se medalhas por um comportamento que achávamos correto, mas depois tentei outras possibilidades de resposta e a consequência era a mesma" (p. J).

Estimulou ainda a reflexão sobre a questão da responsabilidade e responsabilização: "de quem é a responsabilidade desta guerra? Quem assume a responsabilidade destes atos? Acho que neste jogo aprendemos a importância da responsabilidade pela sua ausência" (p. S).

Os/as participantes referem ainda que os dilemas com os quais são confrontados no jogo se encontram relacionados com a realidade fora do jogo e que este jogo dificulta a dicotomização de comportamentos morais: "era um mecanismo eficiente para construir diálogos, mas, por outro lado, nós nunca sabemos se há comportamentos certos ou errados, não temos respostas certas ou erradas" (p. N); "isso permite-nos compreender os dois lados. Não olhar só para um lado e julgar” (p. RB).

\section{Categoria interação social}

Os dados sugerem que a opção de jogo em pares foi avaliada de forma positiva, favorecendo a criação de relações e a partilha de tarefas e o sentimento de controlo sobre o videojogo: "convém estar em pares. Se ficar sozinha sem saber as linhas orientadoras, fico perdida, sem motivação... não me envolvo na história" (p. I); "O jogo em pares sugere favorecer a colaboração e apoio na tomada de decisões: "mesmo quando corre mal, a pessoa está acompanhada" (p. M).

Adicionalmente, jogar em pares permite uma maior facilidade na coordenação e concretização de tarefas em simultâneo: "Se estiverem dois é mais fácil a divisão de tarefas: um concentra-se mais na ação mecânica e o outro no plano e estratégia" (p. J).

Ao mesmo tempo, os/as participantes referem que o videojogo Ihes permitiu desenvolver competências de negociação, momentos propícios para trabalhar relações colaborativas, a par do desenvolvimento de competências relacionais como a ajuda, empatia, tomada de perspetiva do Outro: "a identificação com a experiencia do personagem foi o que mais me ficou deste jogo, (p. E).

A gestão de tempo e de várias tarefas em simultâneo foram igualmente referidas: "acho que era intencional do próprio jogo, colocarem-nos nesta situação de não conseguirmos fazer duas coisas e falharmos" (p. O).

Assim como a identificação e gestão de emoções e auto-conhecimento: "senti-me mais emocional depois de jogar este jogo" (p. G); "tive de lidar com sentimentos de frustração. Acho que nos estavam a obrigar a desistir de algo (p. P).

Embora o carácter narrativo do jogo tenha favorecido o envolvimento com a vida da personagem, alguns/as participantes advogam que o jogo tem demasiada informação 
de texto: "o jogo tem muito diálogo. Há muito a ler e pouca ação, pouco para fazer" (p. N).

Ao mesmo tempo, esta narratividade parece favorecer a reflexão sobre identidades e papéis sociais (pai, marido, colega de trabalho) e sobre as relações familiares e profissionais de um homem adulto.

Reflexões sobre diferenças culturais, estereótipos e preconceitos foram também indicadas, a par de reflexões sobre relações de poder, manipulação e controlo dentro e fora do jogo. Relativamente a questões de poder, o videojogo desafiou os/as participantes a refletir sobre a questão do controlo, interpretada como tentativa de manipulação: "não me agradou o facto de saber que o jogo tinha mais poder que eu e eu estar sempre a ser controlada pelo jogo" (p. l).

\section{Categoria ação cívica e política}

Os dados sugerem que o videojogo motiva para o envolvimento em ações cívicas e políticas fora do jogo, e que os jogadores/as se mostram mais predispostos a agir perante questões cívicas significativas: "o jogo faz com que as pessoas sejam sensibilizadas para questões do foro politico, em termos de consciencialização enquanto adultos" (p. JR); "Este jogo faz com que as pessoas normalmente se posicionem... ou para mudar ou para se mudarem a si próprias, porque começa por aí, não é mudar os outros..." (p. J).

Os/as participantes refletiram ainda sobre o facto da personagem principal se constituir como a personificação da anomia, da alienação e da ausência de consciência "um funcionário que cumpre as normas do seu estado (p. L) e refletiram sobre o papel de um/a cidadão/cidadã, na tensão entre a obediência e a transgressão.

\section{Categoria criatividade social}

Os dados sugerem, por fim, o surgimento de uma categoria emergente, que diz respeito à criatividade e criação de narrativas diversas. Alguns/as jogadores/as percecionaram este videojogo, associando-o à criação artística da narrativa de um filme: "é como um guião de um filme" (p. RD); "parecia uma coisa mais misteriosa e artística, não sei adjetivar" (p. A).

Parece estar patente, de forma constante, a possibilidade de criação, quer da personagem e da narrativa: "o objetivo do jogo é a construção da personagem" (p. J); "a história está a ser criada por nós", quer da realidade: "mostra-nos que existem sempre várias alternativas” (p. J). O videojogo parece favorecer, desta forma, a identificação de possibilidades alternativas de representar, perspetivar e criar a realidade e a vida, pelo que pode favorecer o emergir de capacidades criativas, pela combinação destas diferentes alternativas, com potencial para trazer respostas divergentes a questões sociais e políticas. 
De facto, este videojogo parece estimular um processo de "humanização", no sentido de uma criação individual e coletiva, de tudo o que possa tornar-nos humanos, pela capacidade de nos colocarmos na perspetiva comum e partilhada de outros humanos, com as suas necessidades e vivências: "acho que o objetivo do jogo é humanizar-nos (num trocadilho com o título do jogo- desumano) e não apenas matar os terroristas. É colocar-nos nos pés desta pessoa, tentar resolver os seus problemas. É colocar-nos nesta sociedade, tentar resolver os seus problemas..." (p. RD).

\section{Notas finais}

Como notas finais, podemos indicar que a experiência de jogar o videojogo Unmanned, para os/as participantes do estudo, favoreceu a reflexão e discussão participada sobre aspetos de dimensão cívica e política. Encontramos resultados que, em grande medida, são congruentes com o que tem sido proposto por outros/as autores/ as nesta área.

A história do jogo assim como a sua mecânica favoreceram momentos de tomada de consciência, conhecimento e reflexão crítica sobre o sistema social, político e cívico em associação com a vida real (Connolly et al., 2012; Mitgutsch \& Alvarado, 2012).

Os dados sugerem o potencial educativo deste videojogo para o uso em contexto formal e não formal no ensino superior, com possibilidade de conexões curriculares com as áreas de filosofia, história, ética, cidadania, estudos sociais e políticos, psicologia ou literatura. Para que tal aconteça, há que ter em conta os princípios de uma aprendizagem ativa e experiencial (Becker, 2017; Gee, 2009) e integrá-los em ciclos contínuos de ação e de reflexão, que possibilitem dinâmicas de criação de sentido (Freire, 2014).

A análise dos resultados indicou que este é um videojogo que cria desafios éticos, apresenta dilemas, e que confronta os/as jogadores com a necessidade de se posicionarem, envolvendo-os/as num processo de tomada de decisão, baseado em valores e princípios éticos, num clima de gestão de incerteza e ambiguidade (Schrier, 2015), favorável ao desenvolvimento da criatividade e à geração de novas possibilidades de criação narrativa pessoal (e coletiva) (Frasca, 2001).

Os dados sugerem que a cidadania se aprende com as práticas e os significados experienciados na vida (White, 2013). A partir do videojogo, os/as participantes refletiram sobre diferentes formas de aprender e praticar a cidadania, desde as mais relacionadas com o dever e a obrigação moral, ou o cumprimento de regras sociais, às mais associadas à autonomia e à auto-definição do/a cidadão/ã (Bennett, 2008; Raphael et al., 2010).

Assim, estes dados reforçam o potencial deste videojogo para a aprendizagem da cidadania e participação cívica e política (Frasca, 2001; Bogost, 2006), e dos 
videojogos como estratégias para aprender e educar, de forma crítica e transformativa (Gee, 2009; Becker, 2017).

\section{Agradecimentos}

Este trabalho é apoiado por fundos nacionais, através da Fundação para a Ciência e a Tecnologia, IP, e do Ministério da Ciência, Tecnologia e Ensino Superior (FCT/MCTES), no âmbito do projeto de I\&D JoSeES (ref. ${ }^{a}$ PTDC/MHC-CED/7182/2014); é também apoiado por fundos nacionais, através da FCT/MCTES, e pelo Fundo Social Europeu (FSE), através do Programa Operacional do Capital Humano (POCH) do Portugal 2020, no âmbito de bolsa de doutoramento atribuída pelo Programa Doutoral em Ciências da Educação da Universidade do Porto (ref. ${ }^{a}$ PD/ BD/135578/2018); é ainda apoiado por fundos nacionais através da FCT/MCTES no âmbito do Programa Estratégico do CIIE (ref. ${ }^{a}$ UID/CED/00167/2013).

\section{Referências}

Bogost, I. (2006). Playing politics: Videogames for politics, activism, and advocacy. First Monday.

Barab, S. A., Gresalfi, M., \& Ingram-Goble, A. (2010). Transformational play: Using games to position person, content, and context. Educational Researcher, 39(7), 525-536.

Becker, K. (2017). Choosing and Using Digital Games in the Classroom: A Practical Guide: Springer International Publishing.

Bennett, W. L. (2008). Civic life online: Learning how digital media can engage youth: Mit Press.

Bers, M. U. (2010). Let the games begin: Civic playing on high-tech consoles. Review of General Psychology, 14(2), 147-153. doi:10.1037/a0019490

Castells, M. (2011). The rise of the network society: The information age: Economy, society, and culture (Vol. 1): John Wiley \& Sons.

Chambers, R. (2002). Participatory workshops: a sourcebook of 21 sets of ideas and activities. London: Earthscan.

Connolly, T. M., Boyle, E. A., MacArthur, E., Hainey, T., \& Boyle, J. M. (2012). A systematic literature review of empirical evidence on computer games and serious games. Computers \& Education, 59(2), 661-686. doi:http://dx.doi.org/10.1016/j.compedu.2012.03.004

Flanagan, C. A., Syvertsen, A. K., \& Stout, M. D. (2007). Civic Measurement Models: Tapping Adolescents' Civic Engagement. CIRCLE Working Paper 55. Center for Information and Research on Civic Learning and Engagement (CIRCLE).

Frasca, G. (2001). Videogames of the oppressed: Videogames as a means for critical thinking and debate. (Master), Georgia Institute of Technology Atlanta. Retrieved from http://www.ludology.org/ articles/thesis/FrascaThesisVideogames.pdf

Freire, P. (2014). Pedagogia da esperança: um reencontro com a pedagogia do oprimido. Rio de Janeiro: Editora Paz e Terra. 
Gee, J. P. (2009). Deep learning properties of good digital games Serious games: Mechanisms and effects: Routledge Taylor \& Francis Group.

Huizinga, J. (1971). Homo ludens: o jogo como elemento da cultura (Vol. 4). S. Paulo: Editora Perspectiva.

Jenkins, H., Purushotma, R., Weigel, M., Clinton, K., \& Robison, A. J. (2009). Confronting the challenges of participatory culture: Media education for the 21st century: Mit Press.

Kahne, J., Middaugh, E., \& Evans, C. (2008). The civic potential of video games: occasional paper of the John D. and Catherine T. MacArthur Foundation

Digital Media and Learning Program.

Khaled, R., \& Vasalou, A. (2014). Bridging serious games and participatory design. International Journal of Child-Computer Interaction, 2(2), 93-100.

Könings, K. D., van Zundert, M. J., Brand-Gruwel, S., \& van Merriënboer, J. J. (2007). Participatory design in secondary education: Is it a good idea? Students' and teachers' opinions on its desirability and feasibility. Educational Studies, 33(4), 445-465.

Mitgutsch, K., \& Alvarado, N. (2012). Purposeful by design?: a serious game design assessment framework. Paper presented at the Proceedings of the International Conference on the foundations of digital games.

Oliveira, R., \& Pessoa, T. (2008). Benefícios Cognitivos dos Videojogos: A percepção dos jovens adultos. ZON Digital Games 2008, 48-53.

Pinto, M., \& Ferreira, P. (2017). Use of Videogames in Higher Education in Portugal: a Literature Review. Paper presented at the Conferência Internacional de Tecnologias de Informação e Comunicação na Educação - Challenges 2017, Braga.

Raphael, C., Bachen, C., Lynn, K.-M., Baldwin-Philippi, J., \& McKee, K. A. (2010). Games for civic learning: A conceptual framework and agenda for research and design. Games and culture, 5(2), 199-235.

Schrier, K. (2015). EPIC: A framework for using video games in ethics education. Journal of Moral Education, 44(4), 393-424.

Squire, K. (2011). Video Games and Learning: Teaching and Participatory Culture in the Digital Age. Technology, Education--Connections (the TEC Series): ERIC.

Stevenson, J. (2011). A Framework for Classification and Criticism of Ethical Games Designing Games for Ethics: Models, Techniques and Frameworks (pp. 36-55). Hershey, PA, USA: IGI Global.

White, M. (2013). Higher education and problems of citizenship formation. Journal of Philosophy of Education, 47(1), 112-127. 


\section{Lucinda Saldanha}

Mestre em Psicologia pela Universidade do Porto e em Estudos Artísticos pela Universidade de Coimbra, é atualmente doutoranda no Programa Doutoral em Ciências da Educação da Faculdade de Psicologia e Ciências da Educação da Universidade do Porto, financiado pela Fundação para a Ciência e a Tecnologia, IP. No âmbito deste programa, foi-lhe atribuída bolsa de doutoramento com a ref. ${ }^{a}$ PD/BD/135578/2018. E-mail: lucindasaldanha@fpce.up.pt

ORCID: 0000-0001-5874-0915

Marta Pinto

Doutorada em Multimédia em Educação pela Universidade de Aveiro, é atualmente Investigadora em Pós-Doutoramento no Centro de Investigação e Intervenção Educativas (CIIE) da Faculdade de Psicologia e Ciências da Educação da Universidade do Porto, com bolsa de pós-doutoramento financiada pela FCT (ref. ${ }^{a}$ SFRH/BPD/116440/2016).

E-mail: mpinto@fpce.up.pt ORCID: 0000-0001-8271-2818

Pedro D. Ferreira

Professor Auxiliar na Faculdade de Psicologia e de Ciências da Educação da Universidade do Porto e Investigador do Centro de Investigação e Intervenção Educativas (CIIE).

Coordena o projecto JoSeES - Jogos Sérios no Ensino Superior: Impactos, Experiências e Expectativas (FCT - PTDC/MHC-CED/7182/2014).

E-mail: pferreira@fpce.up.pt ORCID: 0000-0002-5010-7397

Correspondência Lucinda Saldanha ClIE-Centro de Investigação e Intervenção Educativas Faculdade de Psicologia e de Ciências da Educação da Universidade do Porto Rua Alfredo Allen 4200-135 Porto Portugal

Data de submissão: Janeiro 2018 Data de avaliação: Março 2018 Data de publicação: Julho 2018 